\title{
EL PENSAMIENTO CRÍTICO Y AUTOCONOCIMIENTO ${ }^{1}$
}

\author{
Fredy Hernán Prieto Galindo \\ Universidad Pedagógica Nacional \\ Bogotá, Colombia \\ fhprietog@pedagogica.edu.co
}

\section{RESUMEN / ABSTRACT}

En este artículo de reflexión se plantea que el concepto de 'pensamiento crítico' formulado por los pensadores del Movimiento de Pensamiento Crítico contiene un presupuesto que mina en gran medida su empresa: el movimiento asume que el pensador crítico desarrolla un autoconocimiento directo y transparente, lo que implicaría cuasi-infalibilidad. En la historia de la filosofía algunos pensadores han defendido esta posibilidad de un autoconocimiento directo e infalible, por ejemplo René Descartes y más recientemente Donald Davidson. Sin embargo, tal conocimiento parece tener unos límites más o menos claros que no son tenidos en cuenta por los pensadores del MPC al formular su noción de pensamiento crítico, y debido a este presupuesto su proyecto filosófico e incluso pedagógico podría quedar sin fundamento.

Palabras clave: pensamiento crítico, autoconocimiento, autoatribuciones infalibles, habilidades del pensamiento, disposiciones, estados mentales.

\section{CRITICAL THINKING AND SELF-KNOWLEDGE}

This paper argues that the concept of 'critical thinking'formulated by the Thinkers of the Critical Thinking Movement contains a gap that greatly undermines its enterprise: the movement asserts that a critical thinker develops a direct and transparent selfknowledge, which would imply quasi-infallibility. In the history of philosophy some thinkers have defended this possibility of direct and infallible self-knowledge, for example René Descartes and more recently Donald Davidson. However, such knowledge seems to have some more or less clear limits that are not taken into account by the thinkers of the MPC to formulate their notion of critical thinking and by this their philosophical and pedagogical project may be unfounded.

KEYWORDS: Critical thinking, self-knowledge, infalible self-atributions, thinking abilitis, dispositions, mental states.

Este artículo de reflexión surge como uno de los resultados del proceso de investigación monográfico de una Maestría en Educación cursada en la Universidad de los Andes. 


\section{Introducción}

R⿴囗十 En nuestras sociedades contemporáneas, la habilidad de pensar críticamente se considera un atributo indispensable para lo que Paul Ricoeur (2006) llama lograr una vida realizada (con y para los otros en instituciones justas), ya que el pensamiento crítico es necesario para la autonomía y esta para lograr una mejor sociedad (Winch 2006). De ello se sigue que la formación de personas críticas ha de ser un objetivo central de los sistemas de educación contemporáneos (Bailin and Siegel 2003; Siegel 1988; Lai 2011), como bien lo reflejan las políticas públicas de educación colombiana² y la literatura contemporánea sobre educación (Isaza 2010).

Ahora bien, aunque no hay un consenso sobre la definición y comprensión del pensamiento crítico (Harvey Siegel 1988; Sharon Bailin 2002; Alec Fisher 2004), las diversas definiciones poseen elementos comunes entre filósofos de la educación, pedagogos y psicólogos ${ }^{3}$ (Lai 2011), todos ellos definiendo el pensamiento crítico en relación con la educación. Principalmente se encuentra un consenso respecto de dos elementos fundamentales del pensamiento crítico: un componente evaluativo, que contiene las habilidades del pensamiento tales como evaluar, analizar, sintetizar, etc., y un componente disposicional, en el que se conjugan diversas características antropológicas como las emociones, carácter, hábitos, etc.

En este artículo reflexionamos sobre la concepción filosófica de pensamiento crítico que propone el Movimiento de Pensamiento Crítico ${ }^{4}$ (en adelante MPC), que según Hilda Difabio ha tenido una fuerte difusión en los países de habla hispana (2005). En particular, el objetivo de este documento es afirmar que dicho concepto de pensamiento crítico (del MPC) contiene un presupuesto que socavaría su proyecto o

2 Bajo el título Los fines de la educación de la Ley General de Educación (1994), el numeral noveno muestra que aspectos como el mejoramiento o progreso social, económico y cultural, y la calidad de vida de las personas se fortalece gracias a la "capacidad crítica, reflexiva y analítica".

3 Con el fin de profundizar en las características particulares en la comprensión del pensamiento crítico según cada una de estas disciplinas, consultar (Lai 2011), (Bailin 2002).

4 Según Sharon Bailin (2002), el MPC contiene una perspectiva cognitivista, que resulta ser uno de los rasgos fundamentales de esta concepción del pensamiento crítico que la distingue de filosofías como la de la Pedagogía Crítica u otros modelos inspirados en diversas filosofías como las de Michel Foucault o Jürgen Habermas. Si bien pueden haber discrepancias sobre los autores que se vinculan al MPC, aquí los caracterizamos por dos rasgos fundamentales: primero, todos ellos conciben el pensamiento crítico a partir de dos elementos básicos, que son las habilidades del pensamiento y las disposiciones; en segundo lugar, se trata de un conjunto de pensadores que no se dedican solamente a la teorización del pensamiento crítico sino a elaborar propuestas prácticas pedagógicas e instrumentos didácticos e investigativos sobre su enseñanza en educación, colegio y universidad. Entre los pensadores más reconocidos de este movimiento encontramos a Stephen Norris, Robert Ennis, John Mac Peck, Harvey Siegel, Alec Fischer, Richard Paul, Peter Facionne y Sharon Bailin. 
al menos limitaría profundamente sus alcances: estos pensadores asumen que los seres humanos nos conocemos directamente y que este autoconocimiento es transparente, y por ende cuasi-infalible, o muy confiable.

De acuerdo a este presupuesto, un pensador crítico conoce sus propios estados mentales 5 (tales como, creencias, razones, prejuicios, sesgos, deseos, etc.) de tal manera que no necesita desarrollar procesos de inferencia y, por ello mismo, su autoconocimiento se hallaría exento de error o, al menos, sería muy confiable. Por ende, cualquier persona puede confiar en su autoconocimiento para juzgar, criticar, sus propios argumentos y decisiones, y ser autónomo. Ahora bien, si en realidad dicho autoconocimiento directo y transparente no fuese posible ¿qué validez tendrían los juicios críticos y qué tan apropiadas serían las decisiones? En última instancia, ¿cuáles serían los alcances y límites de esta noción particular de pensamiento crítico basada en tal noción de autoconocimiento?

Con el fin de defender tal idea, este texto se desarrolla en el siguiente orden: en primer lugar se desplegará brevemente el concepto de pensamiento crítico a partir de sus dos componentes fundamentales: las habilidades del pensamiento y las disposiciones. Luego, se presentará el tema del autoconocimiento, bajo una perspectiva analítica, con el fin de aclarar un poco los límites del autoconocimiento y sus aportes al pensamiento crítico. En la segunda parte se presenta el autoconocimiento a partir del análisis de las propias definiciones y caracterizaciones del pensamiento crítico que proponen los pensadores del MPC. Finalmente se concluye respecto de la tesis expuesta y se presentan algunas posibles consecuencias de esta idea en el ámbito pedagógico.

\section{Concepto de pensamiento crítico del MPC}

\subsection{Habilidades o habilidades y criterios}

Los autores del MPC consideran que el pensamiento crítico se compone fundamentalmente de un conjunto de habilidades y disposiciones que favorecen el proceso de examen de las razones que sustentan una creencia o una decisión (Fisher 2001; Siegel 2010; Norris \& Ennis 1989). En cuanto al primer elemento, estos filósofos dan algunas listas de habilidades de pensamiento. Por ejemplo, "interpretación, análisis, evaluación, inferencia, explicación y autorregulación" (Facione 2013, p. 5). Pero esta lista es demasiado general. Otra lista es la de Robert Ennis y Stephen Norris; aquí un fragmento:

Tomamos el concepto de estado mental en un sentido general, como ha sido usado en la filosofía analítica para referirse a creencias, sensaciones, emociones, deseos, etc. Resulta irrelevante para este artículo la discusión en torno a las diversas problemáticas alrededor de este concepto, o las diversas posturas que han surgido sobre su definición. 


\section{Clasificación elemental}

1. Centrarse en una pregunta

2. Análisis de los argumentos

3. Hacer y responder preguntas que aclaran y desafían Soporte básico

4. Juzgar la credibilidad de una fuente

5. Hacer y juzgar las observaciones (Norris y Ennis 1989, p. 14).

Al comparar las dos listas de habilidades del pensamiento es evidente que la segunda, que es bastante extensa, muestra con detalle las habilidades que un pensador crítico desarrollaría y aplicaría. Este es precisamente uno de los aspectos que se toman de esta teoría para aplicarlo en la enseñanza del pensamiento crítico, pues se planean ejercicios para que los estudiantes desarrollen estas habilidades.

Ahora bien, los filósofos de la CTM piensan que las habilidades pueden desarrollarse de una manera descuidada, superficial o no reflexiva, por lo que Sharon Bailin aclara que el componente de evaluación debe incluir ciertos criterios:

Es la cualidad del pensamiento la que distingue el pensamiento crítico del no crítico, y esta cualidad está determinada por el grado en que el pensamiento satisface las normas y criterios pertinentes. Es, pues, la adhesión a ciertos criterios la característica definitoria del pensamiento crítico (Bailin 2002, pp. 363-364. Traducción personal).

Por lo tanto, el pensamiento crítico no se centra en las capacidades de pensamiento o los procesos mentales, sino que tiene en cuenta el cumplimiento de los principios, criterios normativos, que proceden principalmente de la lógica formal e informal (Siegel 1997, p. 16). Sin embargo, ¿qué se necesita evaluar? Y, ¿cuáles son esos criterios? Harvey Siegel dice:

Al menos, evaluar la fuerza probatoria o normativa de las razones. Es decir, el pensador crítico debe ser capaz de decir si una razón es genuina; si apoya fuerte o débilmente alguna reclamación o acción por la que se ofrece como razón y si debe, sobre la base del motivo en cuestión, aceptar la reclamación o realizar la acción en cuestión (1997, p. 14. Traducción personal).

Para determinar si una razón es genuina o lo suficientemente fuerte como para apoyar una creencia o acción es preciso buscar falacias, contradicciones, suposiciones, etc. Por lo tanto, los criterios para estos casos específicos son dados por la lógica (formal e informal). Por ejemplo, si la gente oye a un político decir que debe evitar votar por otro candidato porque ha engañado a su esposa, conociendo la falacia ad hominem, podría concluir que esta razón no es válida o no tiene la fuerza probatoria para aceptar la tesis, ni el impacto normativo para hacer lo que el político sugiere. En efecto, el punto final de hacer un examen tan lógico es decidir qué creencias y acciones seguir.

La fuerza probatoria y el impacto normativo son los rasgos de las razones que se tiende a valorar más. El primero tiene que ver con la calidad del argumento, y el 
segundo, con el impacto sobre la voluntad para llevar a una persona a ejecutar una acción. En las palabras de Stefaan Cuypers, "el primer componente se ocupa de la propiedad epistémica de las razones mientras que el segundo captura la motivacional" (2004, p. 76. Traducción personal). Así, el impacto normativo es lo que explicaría la relación del argumento con la acción concreta, ya que es lo que 'empuja' o motiva la voluntad para moverse de una manera u otra, para ejecutar las aciones.

Así, el pensamiento crítico no solo está relacionado con la calidad de las razones, sino también con la acción coherente que la persona debe desarrollar. En palabras de Harvey Siegel, "los estudiantes (y la gente en general) son pensadores racionales, razonables o críticos, en la medida en que creen, juzgan y actúan sobre la base de razones (evaluadas completamente)" (2010, p. Traducción personal). Por lo tanto, un pensador crítico tiene que evaluar las razones, decidir y actuar en coherencia con los resultados de esa evaluación. Aquí es evidente que el punto del pensamiento crítico no es solo pensar bien, sino actuar en consecuencia.

Hasta ahora, el enfoque en la lógica es obvio, pero este énfasis en la acción lleva el concepto a otra dimensión aparte de la racionalidad. Dado que una persona puede desarrollar todas las capacidades de pensamiento crítico y cumplir los criterios pertinentes, pero podría no aplicarlos habitualmente ni actuar en consecuencia, la mayoría de los filósofos del MPC (por ejemplo: Siegel 2010, Bailin 2002, Ennis 1993, Norris \& Ennis 1989) piensa que este tipo de comportamiento no puede ser llamado pensamiento crítico. En palabras de Harvey Siegel, "no es suficiente que una persona sea capaz de evaluar adecuadamente las razones; para ser un pensador crítico, ella debe realmente involucrarse [actually engage] en la evaluación competente de la razón, y generalmente estar dispuesta a hacerlo “(1997, p. 3. Traducción personal).

\subsubsection{Disposiciones: más que la razón}

Entonces, junto con las habilidades y los criterios, el pensamiento crítico incluye un conjunto de disposiciones, hábitos mentales y rasgos de carácter, generalmente denominados componente disposicional o espíritu crítico. "Este extiende el ideal más allá de los límites del ámbito cognoscitivo, porque, así entendido, el ideal es el de una persona determinada" (Siegel 2010, pp. 142-143. Traducción personal). El espíritu crítico implica dimensiones humanas como libertad, voluntad, tendencias, hábitos, etc. Aquí un fragmento de la lista de Ennis:

1. Cuidar que sus creencias sean verdaderas, y que sus decisiones sean justificadas; es decir, el cuidado de "pensar correctamente" [get it right] en la medida de lo posible, incluyendo:

a. Buscar hipótesis alternativas, explicaciones, conclusiones, planes, fuentes, etc.; y estar abierto a ellos;

b. Considerar seriamente otros puntos de vista diferentes a los suyos;

c. Tratar de estar bien informado;

d. Aprobar una posición en la medida en que, y solo en la medida en que se justifica por la información que está disponible; 
e. Utilizar sus habilidades de pensamiento crítico (2011, p. 2. Traducción personal).

Todas estas disposiciones muestran un fuerte vínculo con la lógica incluida en el componente evaluativo. Un pensador crítico tiene las habilidades necesarias para razonar adecuadamente, no solo desde una perspectiva lógica, sino también disposicional. Aplica habitualmente su razón, porque es un cierto tipo de persona, que actúa de cierta manera, bajo ciertas disposiciones. El pensamiento crítico se refiere a "realmente ser y actuar críticamente; es decir, ser (apropiadamente) movido a creer y actuar basado en razones "(Siegel 1988, p. 142. Nota al pie 14. Traducción personal). Este énfasis en la persona como un pensador crítico y no solo en ciertas características debe ser considerado cuidadosamente, ya que incluye todas las dimensiones humanas en la comprensión del pensamiento crítico.

Ser un pensador crítico es ser una persona con cierto carácter, más que una persona que solo tiene algunas habilidades y disposiciones. El pensamiento crítico para el MPC no se trata solo de procesos de decisión, ni de acción; es sobre todo ser un cierto tipo de persona. Un pensador crítico es una persona libre que voluntariamente decide y actúa después de un examen cuidadoso de los argumentos de acuerdo con los criterios pertinentes.

El 'espíritu crítico' no es simplemente una lista de disposiciones, sino el carácter mismo de la persona. Poseer las habilidades y disposiciones para creer o actuar críticamente no es suficiente para ser realmente un pensador crítico. Consideremos los fumadores que deciden renunciar al vicio, pero nunca logran su decisión. El objetivo último del pensador crítico no es pensar sino actuar. Según Harvey Siegel (1988, 2010), el pensamiento crítico es en realidad un tipo de persona bien definido, una que es movida por razones. Sin embargo, ¿qué significa? ¿Qué se debe mover por razones? ¿Tal vez dejar de lado todas las dimensiones de los seres humanos y prestar atención solamente a los argumentos? ¿Cuál es el papel de las disposiciones o espíritu crítico y cuál es su relación con la razón?

Harvey Siegel divide el espíritu crítico en tres: disposiciones, actitudes y rasgos de carácter. Aquí algunos ejemplos de cada categoría:

El espíritu crítico, tal como yo uso el término, se refiere a un complejo de disposiciones, actitudes y rasgos de carácter. Incluye disposiciones, por ejemplo, las disposiciones para buscar razones y pruebas en la toma de juicios y para evaluar tales razones cuidadosamente de acuerdo con los principios relevantes de la evaluación de razones. Las actitudes, incluyendo el respeto a la importancia del juicio razonado y de la verdad, y el rechazo de la parcialidad, la arbitrariedad, los alegatos especiales, los deseos ilusorios y otros obstáculos al ejercicio adecuado de la evaluación de la razón y del juicio razonado. Hábitos mentales constantes con estas disposiciones y actitudes, tales como los hábitos de buscar y evaluar las razones, de tomar debidamente en consideración los principios de la evaluación de las razones, de someter las razones al escrutinio crítico y participar en la imparcialidad y la consideración desinteresada de tales razones. Y rasgos de carácter afines con todo esto. Las personas que poseen el espíritu crítico valoran 
un buen razonamiento, y están dispuestas a creer, juzgar y actuar con base en ellos (Siegel 1997, pp. 33-34. Traducción personal).

En términos generales, todos estos componentes del espíritu crítico muestran que el pensamiento crítico es más que una simple lista de cualidades. Se trata de la persona como un todo y no solo algunos episodios o características particulares. A primera vista, parece que el espíritu crítico solo mueve la razón para hacer su trabajo. Parece como si su valor estuviera en su poder para hacer que la razón ejecute sus habilidades. Por ejemplo, en cuanto a las actitudes, tal vez más cercanas a un significado moral, hacen que la gente obedezca a la razón o ejecute lo que la razón propone de ciertas maneras: imparcial, respetuosamente, correctamente.

Las disposiciones son guías para que la razón haga bien su trabajo, es decir, para evaluar apropiadamente los argumentos. Lo importante no es si el espíritu crítico causa o hace que la razón desarrolle sus habilidades, sino que guían a la razón para hacerlo de cierta manera, y sobre todo guíen a la persona según las razones encontradas: mueven apropiadamente al pensador crítico. Por lo tanto, los pensadores críticos no son solo personas bien capacitadas en habilidades de pensamiento. Por el contrario, se mueven por su propio espíritu crítico para valorar buenas razones y actuar en consecuencia. Un pensador crítico actúa según las razones evaluadas porque considera las razones suficientemente fuertes y la acción conveniente.

Respecto del pensamiento crítico, definido a partir de los componentes evaluativo y disposicional, cierto conocimiento de sí mismo es necesario para ser verdaderamente una persona crítica, puesto que tal conocimiento (de las tendencias o inclinaciones personales, de los prejuicios, emociones, razones, etc.) se requiere en el momento de evaluar argumentos o información, so pena de favorecer o desfavorecer una creencia o acción a examinar. En otras palabras, sin un conocimiento de ciertos aspectos personales, las evaluaciones realizadas pueden ser tan sesgadas y parciales que finalmente no habría un pensamiento crítico imparcial sino totalmente sesgado.

Se podría decir que precisamente el objetivo de un pensador crítico es conocer tales estados mentales y que lo logra gracias al pensamiento crítico, bien sea que ya esté desarrollado o que se esté aprendiendo. Pero aquí es donde se halla la posibilidad de estar incluyendo en la comprensión de tal tipo de pensamiento la condición de un conocimiento de sí mismo directo y transparente, es decir, que el pensador crítico logra conocer sus prejuicios, inclinaciones, etc., porque puede conocerse sin dificultades. En este sentido, bien sea que antes de iniciar un ejercicio de pensamiento crítico o precisamente como parte fundamental de este, esta postura se basa en que el pensador crítico puede conocer sus estados mentales con claridad.

Ya que un pensador crítico ha de actuar con base en razones completamente evaluadas, como Harvey Siegel demanda (1988; 1997), si la evaluación de tales razones no contempla los posibles vicios de fondo en el conjunto de creencias y tendencias personales, la conclusión de tal valoración puede resultar tan sesgada como si no hubiese pensamiento crítico en absoluto, con el agravante de que habrá un conjunto de razones o racionalizaciones que la justificarían, cayendo por ende en un autoengaño, justificando así cualquier creencia o acción. 
Entonces, surgen las siguientes inquietudes sobre este supuesto: ¿qué podemos conocer acerca de nosotros mismos con certeza y cuáles son las características de tal autoconocimiento? ¿Cuáles son los límites y alcances del conocimiento de sí mismo? Tales inquietudes se abordarán en la siguiente sección.

\section{Autoconocimiento directo e infalible}

Dentro de su epistemología parece que los autores del MPC asumen que es posible el autoconocimiento directo e infalible; es decir que no se requiere ningún método inferencial para que un individuo conozca sus estados mentales (pensamientos, sensaciones emociones, creencias, etc.); y es infalible o, según propuestas moderadas, casi-infalible, puesto que no es posible que se equivoque sobre aquellos, (casi)siempre los puede conocer tal como ellos son, solo basta un ejercicio de introspección o percepción interna.

Según Liza Skidelsky (2011), alrededor del autoconocimiento o atribución y autoatribución de estados mentales, se han desarrollado diversas corrientes filosóficas, que suelen llamarse Teoría de la Mente, cuya tesis central gira en torno a la asimetría entre la primera y tercera persona en la atribución de sus estados mentales. En palabras de Gilbert Ryle, se piensa que "la mente posee poderes superiores para aprehender sus propios estados y operaciones que los que ella posee para aprehender los hechos del mundo externo" (2009, p. 136. Traducción personal). Según este filósofo, desde Descartes algunos pensadores han establecido que los supuestos estados, episodios o procesos mentales ocurren siempre conscientemente y no pueden ser engañosos. "Si yo pienso, espero, recuerdo, quiero, lamento, escucho un ruido o siento un dolor, yo debo, ipsofacto, conocer que yo lo hago" (Ryle 2009, p. 140. Traducción personal).

Más recientemente, una posición moderada ha sido sostenida, por ejemplo, por Donald Davidson, quien afirma que "rara vez se da el caso en que yo necesite o apele a la evidencia u observación con el fin de averiguar lo que yo creo; normalmente yo sé lo que pienso antes de hablar o actuar" (1987, p. 441). Incluso en esta versión 'débil' del autoconocimiento, es evidente que tal conocer parece poseer una fuerza tal que en circunstancias normales llevaría a confiar fuertemente en los juicios sobre los propios estados mentales.

Ahora bien, según Liza Skidelsky (2011), en general parece haber dos posturas sobre este problema: unas abogan por un acceso epistémico privilegiado a los propios estados mentales y otras invocan la autoridad de primera persona. Sobre el acceso especial, esta pensadora afirma que

estamos, habitualmente, en una mejor posición que otras personas para conocer lo que pensamos, dado que tenemos un método especial para acceder a nuestros propios estados mentales que consiste en una especie de percepción interna. Según esta perspectiva, accedemos a nuestros propios estados mentales porque tenemos un mecanismo cognitivo de monitoreo que produce estados de nivel superior acerca de estados de nivel inferior (Skidelsky 2011, p. 36). 
Así, gracias a cierto mecanismo de monitoreo interno o de percepción interna (nombrada también introspección) podemos conocer inmediatamente nuestros propios estados mentales a medida que ellos van ocurriendo, pues tal introspección es, diríamos, automática, es decir que siempre está activa. Evidentemente, la consecuencia más importante es que los juicios sobre nuestros estados mentales son totalmente fiables o infalibles.

Ahora, la autoridad de primera persona se presenta así:

La idea básica es que la práctica de la auto-atribución está parcialmente constituida por la inmunidad al desafío de la autoridad de la primera persona. Las autoatribuciones poseen una presunción de verdad cuya negación por parte de un interlocutor, en contextos normales (i.e. cuando el auto-atributor no es insincero o insano), sería irrazonable o impropia (Skidelsky 2011, p. 36).

En este caso no se trata de una capacidad especial, sino que cualquier persona en condiciones normales posee plena autoridad respecto de lo que ella misma afirma sobre sus propios estados mentales en un contexto normal. Su autoridad, por tanto, se basa en el hecho de que es totalmente consciente y claro lo que ocurre en su mente, por ende, esta teoría no propone ni exige ningún método o capacidad especial de introspección para acceder a los propios estados mentales. Las condiciones de normalidad para confiar en tal autoconocimiento se reducen a que la persona sea sincera y sana mentalmente.

En última instancia, la diferencia entre la posición por el acceso epistémico privilegiado y la autoridad de primera persona radica en el criterio de verificación del conocimiento; en el primer caso, se trata de cierto mecanismo de monitoreo y en el segundo, simplemente de la autoridad de la persona cuando habla con sinceridad de sus estados mentales. Desde luego, en comparación con la primera versión del autoconocimiento, en la teoría de la autoridad de primera persona, la confianza en las aserciones sobre los estados mentales es mucho más moderada, y por tanto el autoconocimiento no sería infalible, pero en general seguiría siendo muy confiable, lo que, en términos prácticos, conduce a la misma presunción de autoconocimiento fiable a la hora de realizar un examen crítico de los propios argumentos.

Así, prescindiendo de las diferencias entre estas dos posiciones sobre el autoconocimiento, la primera y más importante consecuencia de estas posturas es su presunción de verdad, pues, como afirma Fricker, ambas teorías son "explicaciones alternativas de la fiabilidad de nuestras auto-adscripciones de estados mentales, noinferidas psicológicamente" (citada por Skildesky 2011, p. 38). Se trata, por tanto, de un autoconocimiento fiable de los propios estados mentales en los que cualquier persona puede basarse para realizar sus ejercicios de pensamiento crítico al evaluar acciones o creencias.

Ahora bien, para Efraín Lazos “el autoconocimiento es, en efecto, saber hechos acerca de mí mismo, siempre que sean éstos del tipo relevante, esto es, mental o psicológico, y siempre que nuestro acceso a ellos sea inmediato" (2008, p. 176). En otras palabras, hay ciertas condiciones para que una proposición y su autoatribución cuenten como autoconocimiento: I) la creencia se debe poder expresar en primera persona (yo), por tanto, es consciente; II) debe ser sobre estados mentales o psicológicos 
y no sobre hechos físicos como la estatura o el peso; III) también debe ser inmediata, es decir, no se requiere recabar evidencia, ni de un proceso de inferencia para conocer un estado mental, y por ende no hay un medio por el cual se conoce el estado mental.

Por ejemplo, en este momento tengo un dolor moderado en mi hombro derecho. Esta sensación la conozco inmediatamente, no requiero de un proceso de inferencia racional para identificar este estado mental (dolor), y, por supuesto, puedo formular la siguiente proposición actitudinal: me duele el hombro derecho. Fácilmente se puede ver que cumple las tres condiciones expuestas por Efraín Lazos. Pero miremos este otro ejemplo: alguien puede pensar que no es racista y sostiene su creencia en ciertas razones que le parecen suficientemente fuertes: a) saluda cordialmente a sus vecinos de color; b) comprende que son una cultura diferente y por ello se comportan de modo muy diferente a él, incluso pareciéndole irritantes en ocasiones; c) tiene amigos cercanos que son de color. Podríamos pensar que sus razones cumplen las condiciones señaladas y por tanto aquí habría autoconocimiento.

Pero ¿qué pasa si aparte de las razones esgrimidas por esta persona para creer que no es racista, otra persona ha visto que siempre que puede evade o mantiene al mínimo su contacto con personas de color, y además, que algunas de sus afirmaciones sobre ellos suelen ser despectivas y que, finalmente, los que llama amigos cercanos son solo compañeros de trabajo? ¿Diríamos que en realidad se conoce? ¿O más bien se autoengaña?

Alex Madva (2012) enumera múltiples investigaciones que reafirman esta posibilidad del autoengaño, bajo el término psicológico de actitudes implícitas (implicit attitudes $)^{6}$. Por ejemplo, menciona la investigación de Uhlmann ${ }^{7}$, en la que se concluye que "los juicios sobre las herramientas necesarias para ser un jefe de policía exitoso coinciden con el sentimiento interior [match feeling] de que un hombre es mejor [better suited] que una mujer para este trabajo", aunque la mujer tenga una hoja de vida mejor que la de un hombre. Así mismo, otro estudio concluye que "es menos probable que un doctor le dé la misma calidad de tratamiento médico a pacientes de color que a pacientes blancos" (Madva 2012, p. 16, traducción personal), aunque crea que es imparcial en sus juicios médicos.

6 En psicología se usa el término 'actitud implícita' para referirse a las asociaciones automáticas entre grupos sociales (tales como la raza, el género y la orientacción sexual) y el comportamiento o característias positivas o negativas. Las actitudes implícitas contrastan con las actitudes explícitas que se expresan particularmente en los juicios autorreflexivos de un individuo (Madva 2012, p. 2).

7 Uhlmann, E.L., and Cohen, G.L. 2005: Constructed criteria: Redefining merit to justify discrimination. Psychological Science 16, 474-480.

8 Green, A.R., Carney, D.R., Pallin, D.J., Ngo, L.H., Raymond, K.L., Iezzoni, L., et al. 2007 : Implicit bias among physicians and its prediction of thrombolysis decisions for black and white patients. Journal of General Internal Medicine 22, 1231-1238. 
Esta investigadora resume las posiciones de filósofos como Davidson, Shoemaker y Velleman, afirmando que "las actitudes implícitas obviamente no son creencias, ya que fallan al cumplir ciertos criterios cognitivos sofisticados, tales como ser verificables por medio de evidencia, ser disponibles para una reflexión consciente o ser asimilables a otras creencias, deseos e intenciones" (Madva 2012, 17, traducción personal). De esta cita, quisiera concentrar la atención en el hecho de que dichas actitudes implícitas no son como las creencias, pues no están disponibles para una reflexión consciente.

Como bien recordará el lector, más arriba mencionábamos que algunas de las condiciones del autoconocimiento implicaban la posibilidad de que los estados mentales fuesen conscientes. Así mismo, aunque no era necesariamente debido a la primera condición, sería posible verificar dichos estados mentales ante la evidencia disponible. Estas dos condiciones resultan no satisfechas en el caso de las actitudes implícitas (prejuicios, sesgos, tendencias, etc.), lo que lleva necesariamente a negar la posibilidad de su autoconocimiento, y más aún en nuestro caso, sobre la posibilidad de su conocimiento inmediato y fiable.

Así, pues, habría que aceptar que hay algunos estados mentales que no se pueden conocer directa e inmediatamente, tales como los prejuicios, sesgos, tendencias o, en general, las actitudes implícitas. A diferencia de los dolores, los gustos personales, las sensaciones o pensamientos, las actitudes implícitas mencionadas suelen estar escondidas u ocultas bajo nuestros pensamientos y acciones. En ocasiones debido a la censura social llegamos a construir imágenes de nosotros mismos que responden a lo que la misma sociedad valora y nos conduce a esconder estos otros estados mentales que no son aceptados socialmente. Se trata del autoengaño que expone Chaïm Perelman cuando afirma, siguiendo al psicoanálisis, que "uno se puede engañar a sí mismo y que las razones que se dan pueden ser solamente racionalizaciones” (2007, p. 63).

Por ende, si bien podemos aceptar que hay ciertos conocimientos directos y casi-infalibles de los estados mentales, como los pensamientos, es preciso tener en cuenta que hay otros conocimientos de estados mentales que no son ni directos ni infalibles y que fácilmente podríamos caer en autoengaños al reforzarlos con razones sobre nosotros mismos.

Desde luego, si resulta cierto que los pensadores del MPC se basan o incluyen una posición de autoconocimiento directo infalible, es preciso saber sobre qué tipo de estados mentales tal posición resulta válida y si son estos los que propone este grupo de pensadores. ¿Acaso se trata solamente de examinar las razones y creencias conscientes que llevan a sostener X creencia o acción? O, ¿los pensadores del MPC exigen un examen de otros estados mentales? Además, ¿qué tipo de autoconocimiento se encuentra en la teoría del MPC? ¿Tal posición sobre el autoconocimiento minaría o evidenciaría una falencia en la teoría del MPC sobre el pensamiento crítico? 


\section{El autoconocimiento en algunos autores del MPC}

A nuestro modo de ver, la teoría del pensamiento crítico elaborada por el MPC asume o presupone el autoconocimiento inmediato e infalible; de hecho sería una base fundamental para su teoría y por ello lo proponen como un elemento necesario del pensador crítico, aunque lo denominen con varios términos. Por ejemplo, Alec Fisher lo nombra como metacognición y la define como "reflexionar en el pensamiento que usted lleva cuando llega a una decisión"; y añade que "la manera de mejorarla [la reflexión] es pensar en cómo es que usted lo hace habitualmente" (2004, p. 241. Traducción personal).

Por otro lado, bajo el nombre de autoexamen (self-examination), los pensadores del proyecto Delphi afirman que el pensador crítico puede "reflexionar sobre el razonamiento de uno mismo y verificar tanto los resultados producidos como la correcta aplicación y ejecución de las habilidades cognitivas relacionadas" (Facione 1990, pp. 10-11. Traducción personal); además de la revisión de sus razonamientos, un pensador crítico puede "hacer una autoevaluación metacognitiva objetiva y deliberativa [thoughtful] de las opiniones de uno mismo y las razones para sostenerlas" (Facione 1990, pp. 10-11. Traducción personal).

Harvey Siegel, otro pensador altamente influyente del MPC, no toma un nombre determinado para el autoconocimiento, pero lo incluye cuando afirma claramente que "un pensador crítico es capaz de reconocer un pensamiento basado en prejuicios y puede trabajar por erradicarlo" (1997, p. 93. Traducción personal). Y con mayor entusiasmo aún, lo reafirma Peter Facione al sostener: "Uno puede examinar y corregir una inferencia que ha obtenido. Uno puede revisar y reformular una de sus explicaciones, uno puede incluso examinar y corregir su habilidad de examinarse y corregirse a sí mismo" (1992, p. 7. Traducción personal).

Para estos pensadores es claro que podemos acceder a nuestros pensamientos y procesos de pensamiento inmediatamente, pues simplemente hay que pensar en cómo pensamos. Si contamos las condiciones dadas en el acápite anterior, encontramos que se cumplen cabalmente: efectivamente al pensar en cómo he pensado o llegado a una decisión, puedo formular las proposiciones en primera persona (yo) y ello implica que soy consciente; además, no sugieren que sea necesario ningún proceso de inferencia, sino que hay un acceso directo a los pensamientos y procesos mentales, pues solo requiere reflexionar o pensar en lo que se hace cuando piensa o toma decisiones. Parafraseando a Lazos, se trata entonces de una autoconciencia inmediata de nuestros propios estados mentales (2008). Esta autoconsciencia inmediata es evidente en la siguiente cita de los pensadores del proyecto Delphi cuando afirman que un pensador crítico puede

monitorear auto-conscientemente las actividades cognitivas, los elementos usados en esas actividades y los resultados producidos, particularmente al aplicar las habilidades en el análisis y evaluación de los juicios inferenciales de uno mismo con el fin de cuestionar, confirmar, validar o corregir los razonamientos o resultados (Facione 1990, p. 9, traducción personal). 
Por tanto, se sigue que bajo la perspectiva del MPC los seres humanos podemos identificar inmediata y plenamente las razones o los procesos mentales por los cuales afirmamos una creencia o decidimos ejecutar cierta acción. Desde esta perspectiva, los pensadores del MPC aceptan el autoconocimiento y lo incluyen dentro de las habilidades del pensamiento necesarias para el pensador crítico. En consecuencia, estos pensadores solamente consideran necesaria la evaluación de razones para decidir qué creencias seguir y qué acciones desarrollar y para ello hay que "tratar de corregir las debilidades pensando autoconscientemente sobre cómo usted desarrollará la siguiente decisión" (Fischer 2004, p. 241. Traducción personal).

Por otro lado, parece que estos pensadores se adhieren a la primera versión del autoconocimiento, es decir, al acceso privilegiado que menciona Liza Skildesky, pues incluyen explícitamente algunos de los elementos propios de tal teoría, como el monitoreo o mecanismo de introspección que permite el acceso directo, y, por ende, sería necesario pensar en cómo pensamos, según Fischer, o "reflexionar sobre el razonamiento de uno mismo", según el proyecto Delphi. Diríamos que no se trata de la teoría de la autoridad de primera persona, ya que precisamente lo que piden estos pensadores es "hacer una autoevaluación metacognitiva objetiva y deliberativa (thoughtful) de las opiniones de uno mismo y las razones para sostenerlas" (Facione 1990, p. 10-11. Traducción personal). Por lo tanto, no es suficiente que la persona sea o esté autoconsciente de sus estados mentales, que sea sincera y que esté sana mentalmente. Aunque cumpla estas condiciones ha de revisar sus propios argumentos y creencias.

Ahora bien, el autoconocimiento no es solamente una posición general, sino un supuesto fundamental en la concepción del pensamiento crítico que ha elaborado este movimiento. Tanto en el componente evaluativo (las habilidades del pensamiento y los criterios) y el espíritu crítico (el conjunto de disposiciones) se halla implicado el autoconocimiento aquí enunciado.

En primer lugar, desde el componente evaluativo del pensamiento crítico, Sharon Bailin (2002) afirma que la calidad del pensamiento se determina por el grado con el que el pensamiento cumple los estándares y criterios relevantes (p. 363), que se obtienen de la lógica formal e informal (Siegel 1997, p. 16). Pero ¿cuáles son esos criterios o estándares? Se trata sobre todo de la fuerza probativa y normativa (expuestas arriba).

La fuerza probativa se puede validar, por ejemplo, bajo el criterio de las falacias que aporta la lógica informal; desde luego, si se encuentra una falacia en un argumento, esto es suficiente para que tal razón no sea válida para sostener una idea ni una acción. Aquí, el autoconocimiento se requiere para determinar cuáles son las razones que nos mueven para creer algo o para desarrollar una acción determinada. Solamente bajo este acceso a nosotros mismos podemos identificarlas y evaluarlas bajo los criterios propuestos.

Por ejemplo, si una persona ha decidido negar una oportunidad laboral a una mujer, bajo la razón de que su curriculum vitae no muestra que posea las capacidades requeridas para el trabajo, resultaría que sus razones son válidas en cuanto uno de criterios más fuertes para otorgar un empleo es que el candidato posea las características 
o perfil necesarios. Empero, ¿cómo saber si su valoración se halló influenciada por un prejuicio hacia las mujeres, que ellas no son tan inteligentes o fuertes como los hombres? Si este prejuicio no es consciente, inmediato y por ende el entrevistador no lo conoce, su decisión no es la de un pensador crítico.

Respecto de las habilidades del pensamiento, tales como analizar y evaluar razones, segundo elemento del componente evaluativo, es claro que también se requeriría del autoconocimiento, al menos para saber cuáles habilidades ya se poseen y para saber qué tan desarrolladas están; igualmente se requiere para determinar sus posibles fallas y si se están corrigiendo. En general se trata de un seguimiento del proceso de adquisición y desarrollo de tales habilidades. ¿Acaso una persona sabe inmediatamente que posee tales y tales procesos cognitivos y que además están en tal o tal nivel de desarrollo? Si bien es cierto que estos conocimientos se pueden saber, no parece ser posible que se adquieran directamente, sino por medio de test psicológicos diseñados para tal fin, o por medio de un tercero que conozca los rasgos o acciones específicas que corresponden a una habilidad y sus niveles de desarrollo. De igual forma, un autoconocimiento de tales aspectos se podría dar bajo la revisión de las propias acciones, comparándolas con las que supuestamente satisfacen una habilidad determinada. En ninguno de estos casos el conocimiento sería inmediato.

Respecto del componente disposicional, el presupuesto del autoconocimiento se encuentra, por ejemplo, cuando los pensadores del proyecto Delphi afirman que una de las disposiciones "es la honestidad en enfrentar las inclinaciones, prejuicios, estereotipos, tendencias egocéntricas o sociocéntricas" (Facione 1990, p. 2); o cuando Sharon Bailin y Harvey Siegel (2002) nombran el pensamiento ecuánime (fair-mindness), el pensamiento independiente; es evidente que para lograr este tipo de disposiciones es necesario acceder a los prejuicios, inclinaciones, etc., para poder ser honesto y ecuánime en su reconocimiento.

Desarrollar estas disposiciones implica poder acceder a sí mismos con el fin de identificar estos elementos que oscurecerían e invalidarían las razones para creer o actuar. ¿De qué otra manera se podría hacer esto, de qué manera podría cualquier persona aceptar tales aspectos si no pudiese adentrarse en sí mismo y encontrarlas? Los pensadores del MPC presuponen el autoconocimiento directo y transparente y lo asimilan como 'habilidad' fundamental del pensador crítico.

Tal vez las personas podamos acceder directamente a algunas de nuestras experiencias, como a nuestras preferencias o gustos, nuestras penas y dolores. Así, yo sé que en determinado momento prefiero o preferí una bebida fría a una bebida caliente o sé que siempre prefiero el helado de fresa al de chocolate; y también sé inmediatamente que me duele una muela o la cabeza, o que en este momento estoy pensando en algún asunto. Empero, reconocer las propias tendencias, prejuicios, inclinaciones, etc. requiere más que simplemente pensar o recordar lo que está(ba) pasando en determinado momento o pensar en las razones que motivaron una creencia o acción. El problema que surge es sobre el conocimiento de esta clase de estados mentales que podrían oscurecer un examen de razones. ¿Acaso los prejuicios, sesgos y tendencias personales son explícitos, se pueden conocer inmediatamente? 
Harvey Siegel afirma que los prejuicios "transgreden los cánones de la evaluación racional; algunas veces sobregeneralizan ilícitamente, rechazan o ignoran evidencia y, ciertamente, se protege a sí mismos de evidencia contraria” (Siegel 1997, p. 92, traducción personal). Tales faltas se deben precisamente a que los prejuicios no son conscientes a los individuos, sino que permanecen ocultos o subyacentes al pensamiento y las acciones. Así, estos estados mentales como los prejuicios, sesgos o inclinaciones personales no se podrían identificar inmediatamente a pesar de un mecanismo de monitoreo constante y tampoco podríamos confiar en la autoridad de primera persona. Diríamos, más bien, que probablemente estos estados mentales podrían ser identificados más fácilmente por otra persona con la que continuamente convivimos y que observa nuestras acciones y palabras.

Hasta este punto se ha mostrado que los pensadores del MPC ciertamente incluyen una noción de autoconocimiento directo y casi-infalible y es uno de los fundamentos de su concepción del pensamiento crítico. Tal asunción o presupuesto no se refiere solamente a los estados mentales autoconscientes, como las razones y creencias, sino que va más allá al incluir los prejuicios, sesgos e inclinaciones, que "se protegen a sí mismos de evidencia contraria” (Siegel 1997, p. 92, traducción personal), lo que lleva a pensar en el autoengaño y en los límites y validez de una noción de pensamiento crítico que se basa en la noción de autoconocimiento expuesta aquí.

Entonces, y este es el problema de asumir el autoconocimiento, ¿qué tan válida puede ser una creencia o decisión tomada con base en un conjunto de razones que tal vez son simplemente una racionalización, un autoengaño? Pero más grave aún ¿esta posibilidad del autoengaño quiere decir que no es posible cierto autoconocimiento y por ende el pensamiento crítico?

Los filósofos del MPC, como se mostró más arriba, asumen que cada persona puede efectivamente entrar en su propia mente, en su 'naturaleza' y autoconocerse, detectar sus creencias, prejuicios, tendencias, etc. solamente al reflexionar cómo es que cada persona piensa o bajo qué razones actúa en cierto momento, identificar lo que realmente influenció la creencia o la acción. Si bien se podría aceptar que sí hay ciertos conocimientos directos y transparentes a las personas, como las sensaciones corporales, también es aceptado que hay conocimientos inaccesibles directamente, y por el contrario, podemos caer en el autoengaño.

\section{Conclusiones}

Los pensadores del MPC basan su teoría en la posibilidad de un autoconocimiento directo y casi-infalible. Ser una persona crítica implica, al menos para estos pensadores, poder criticar a partir de juicios bien razonados y examinados, es decir, poder identificar las razones o creencias y los propios sesgos o condicionamientos que pueden limitar el ejercicio de la crítica. Se trata de un autoconocimiento de las razones que cimentan un juicio crítico particular, pero más profundamente, de los sesgos, inclinaciones, prejuicios o, en términos generales, actitudes implícitas. Sin embargo, como se ha 
visto en el texto, tal autoconocimiento directo no es posible para estos elementos sino para estados mentales conscientes como los pensamientos o sensaciones. Ahora bien, el desarrollo del pensamiento crítico que no contemple el autoconocimiento de estos elementos, caería bajo la limitación de realizar un ejercicio de crítica sobre las razones y creencias conscientes, pero no sobre las actitudes implícitas que también influyen en las decisiones y la acción. Por ende, sería preciso reflexionar sobre el estatuto ontológico de estos aspectos y cómo ayuda el pensamiento crítico a su conocimiento o cómo podrían conocerse.

Si el autoconocimiento es falible ${ }^{9}$, como se ha mostrado por algunos filósofos bajo el título de autoengaño, entonces nuestros juicios críticos estarían parcializados o viciados de raíz. En última instancia, no seríamos más críticos que el que no examina sus propias creencias y actúa parcialmente basado en sus prejuicios o creencias erróneas. Por ende, el problema del autoconocimiento en el pensamiento crítico es la posibilidad del autoengaño, pues creyendo que conocemos nuestros prejuicios y sesgos evaluamos nuestros pensamientos y acciones y las aprobamos o rechazamos cuando en realidad es probable que estemos engañados sobre tales prejuicios o sesgos.

Desde un punto de vista pedagógico, podríamos pensar que al formar estudiantes con esta noción de pensamiento crítico y su concepción implícita de autoconocimiento, formamos personas que basados en su autoengaño no aceptan críticas sobre sus pensamientos o acciones, pues creen que ellos ya las han evaluado teniendo en cuenta sus prejuicios y sesgos. Suponiendo que sean de mente abierta y acepten la posibilidad de que estén errados, se disponen a examinar sus acciones y razonamientos, pero nuevamente al revisarlos se basarán en su autoengaño y no concluirán otra cosas diferente de lo que ya han dicho o hecho.

En este sentido, a pesar de su disposición de mentalidad abierta, siguen defendiendo su posición, lo que parecería ser una contradicción performativa de los objetivos del MPC, puesto que al buscar una persona crítica, de mente abierta, el autoengaño lleva a formar personas más decididas en sus posiciones. En otras palabras, las consecuencias de esta concepción de pensamiento crítico y su asunción de tal autoconocimiento no se limitarían simplemente al hecho de la ausencia de un pensamiento crítico bien

$9 \quad$ Al reafirmar la falibilidad del autoconocimiento no se está tratando una tesis epistemológica pesimista que deseche la posibilidad del conocimiento de las actitudes implícitas ni del pensamiento crítico como herramienta de mejoramiento de la vida. Por el contrario, lo que se desea resaltar es un peligro latente en esta teoría a causa de la falencia aquí denunciada. Ahora bien, aceptar que el autoconocimiento directo y transparente no llega a las actitudes implícitas nos lleva a una propuesta, que en términos ricoeurianos, implica la búsqueda de una nueva manera de conocimiento de sí mismo, alejada de las pretensiones autofundacionalistas, como las de Descartes y Husserl, y en el otro extremo, de las filosofías de la sospecha, como la de Nietzsche. Se trataría de una hermenéutica de sí, una manera diferente de concebir el conocimiento de sí, que Paul Ricoeur (1996) propone como atestación, junto a una nueva manera de ser o hacer-se, que corresponde a la concepción que el pensador francés propone para la Phrónesis aristótelica. 
fundamentado y no viciado, sino que llegarían hasta provocar tal contradicción, pues al buscar la formación de personas críticas se logra formar personas autoengañadas sobre sus propios procesos de pensamiento crítico, creencias, sesgos, etc. Forman personas ingenuas que creen que actúan críticamente cuando en realidad se dejan influenciar por prejuicios o creencias que ya poseen o que otros logran poner en sus mentes. En última instancia esta noción de pensamiento crítico basada en la noción expuesta de autoconocimiento podría constituirse en una herramienta de manipulación.

Desde luego, la consecuencia que acabamos se señalar se basa en una concepción solipsista del autoconocimiento, enraizada en la introspección como mecanismo de autoconocimiento. Tal introspección implicaría que el autoconocimiento es o puede ser un logro de la conciencia aislada. Por ende, aparece un solipsismo epistemológico que en la actualidad no es aceptado, pues no solo se admite la injerencia de la alteridad en la vida humana, sino que se considera como dimensión fundamental de lo humano y del conocimiento de sí mismo (Ricoeur 1996); somos quienes somos gracias, por y para los otros. Incluso el asceta que vive en el bosque aislado de una comunidad humana, para ser la persona que es, para pensar como lo hace, primero ha debido crecer en medio de una comunidad humana en la que aprendió todo lo que sabe, como su propia lengua, costumbres y creencias, y que, por supuesto, influyó en la formación de su propia personalidad. Además, esta concepción reforzaría una comprensión inadecuada de la autonomía (solipsista), como si ser autónomo implicara la exclusión de la palabra u opinión del otro del conjunto de razones que sustentarían una decisión.

En términos generales, nos atrevemos a pensar que el MPC fundamenta su concepto de pensamiento crítico en una antropología que sobrevalora el poder de la dimensión teórica de la razón. Esta confianza en la razón, herencia de la tradición ilustrada que Harvey Siegel reconoce explícitamente (cf. 1997), podría explicar el porqué de su noción implícita de autoconocimiento. Ahora bien, a pesar de la estrecha relación que establecen estos pensadores entre el pensamiento y la acción, los filósofos del MPC asumen que el elemento epistemológico, es decir, la lógica o coherencia interna de los argumentos que sustentan una acción o creencia, es el elemento más importante para la formación de personas críticas y por ende omiten la reflexión sobre el autoconocimiento y sobre la acción misma. Asumir que la dimensión epistemológica es la más importante en cuanto al pensamiento crítico, es decir, la evaluación de las razones que la soportan, querría decir que los seres humanos efectivamente podemos controlar toda nuestra 'naturaleza' por medio de la razón, controlar nuestras emociones, sentimientos, cuerpo, etc., e incluso las condiciones del medio en el que estamos, nuestro contexto socio-cultural.

\section{Referencias bibliográficas}

Bailin, Sh. (2002), "Critical Thinking and Science Education”, in Science \& Education 11: 361-375, Kluwer Academic Publishers. Printed in the Netherlands.

Boghossian, P. (2012), "Critical Thinking and Constructivism: Mambo Dog Fish to the Banana Patch", Journal of Philosophy of Education, Vol. 46, № 1. 
Cuypers, S. (2004). "Critical thinking, autonomy and practical reason". Journal of philosophy of education 38(1), 75-90.

Davidson, Donald (1987), “Knowing One's Own Mind”, Proceedings and Addresses of the American Philosophical Association, Vol. 60, № 3 (Enero, 1987), pp. 441-458.

Difabio, H. (2005), "El Critical thinking movement y la educación intelectual”, in Estudios sobre Educación, Universidad de Navarra 9, 167-187. http://dspace. unav.es/dspace/bitstream/10171/8919/1/NE.PDF.

Doughty, H. (2007), "Review Essay. The Limits of Critical Thinking”, The Innovation Journal: The Public Sector Innovation Journal, Volume 11 (3), article 11. http://www.innovation.cc/book-reviews/doughty11essay.pdf.

Ennis, R. (1993), "Teaching for higher order thinking”, Theory and practice, Vol 32, Number 3.

Facione, P. A. (1990), Critical Thinking: A Statement of Expert Consensus for Purposes of Educational Assessment and Instruction.

(1997), Critical thinking: What it is and why it counts. Millbrae, CA: California Academic Press.

(2013), Critical thinking: what it is and why it counts. Pearson education. https://docs.google.com/viewer? $\mathrm{a}=\mathrm{v} \& \mathrm{q}=$ cache:Cai9Xa4LAjsJ:www. insightassessment.com/pdf_files/what\%26why2006.pdf $+(2013)+\% 22$ Critica 1+thinking: + what $+\mathrm{it}+\mathrm{is}+$ and + what $+\mathrm{it}+$ counts $\% 22 \&$ hl $=$ es $\& \mathrm{gl}=$ co\&pid $=\mathrm{bl} \& \mathrm{sr}$ cid=ADGEESjsAXrVIVSbsREspCrS5wx_7llsOt_hLx9LQ18fdoHTEKKKNLHLYiY_AapIqr12CS37e-Q8AyZyAcS9GihGN97aI2Y_CIUo_VwK-g9rdCaZKh-j-C9UTbRS3CnoE2X0YmPdo\&sig=AHIEtbS82zvyJnnm7ZLcIad G8I73uO_OSw.

Fisher, A. (2004), Critical thinking. An introduction. Cambridge.

Green, A.R., Carney, D.R., Pallin, D.J., Ngo, L.H., Raymond, K.L., Iezzoni, L., et al. (2007), "Implicit bias among physicians and its prediction of thrombolysis decisions for black and white patients". Journal of General Internal Medicine 22, 1231-1238.

Isaza, R. (2012), Conference: "El Pensamiento Crítico en la Ley de Educación Superior", Tercer foro sobre la Reforma de la Educación Superior en la Universidad del Rosario. http://www.urosario.edu.co/urosario_files/a1/ a1381280-6cbc-4321-9f8d-c0abf238db77.pdf.

Jaffro, L. (2012), "La conception ricoeurienne de la raison pratique. Dialectique ou éclectique?”, Études Ricoeuriennes / RicoeurStudies, Vol 3, № 1, pp. 156-171.

Khonde, N. (2005), La sagesse pratique. Frankfurt: Peter Lang.

Lai, E. (2011), “Critical Thinking: A Literature Review”. Research Report. Pearson education. http://www.pearsonassessments.com/hai/images/tmrs/Critical ThinkingReviewFINAL.pdf.

Lazos, E. (2008), “Autoconocimiento: una idea tensa", Diánoia, volumen LIII, número 61 (noviembre 2008): pp. 169-188. 
MacLaren, P. (1994), "Foreword: Critical thinking as a political project", Walters, $K$. re-thinking reason. State University on New York Press.

Madva, A. (2012), "The Hidden Mechanisms of Prejudice: Implicit Bias \& Interpersonal Fluency". http://academiccommons.columbia.edu/catalog/ac\%3 A153250

MEN. (1994), "Ley General de Educación”. http://www.oei.es/quipu/colombia/ Ley_115_1994.pdf.

Norris, S. P. \& Ennis, R. H. (1989), Evaluating critical thinking. Pacific Grove, CA: Midwest Publications.

Perelman, Ch. (2007), El imperio retórico. Bogotá: Norma.

Ricoeur, P. (2006), Sí mismo como Otro. Madrid: Siglo XXI.

Ryle, G. (2009), The concept of mind. London: Routledge.

Santamaría, V. (2009), “Introducción”, en “Autoengaño y problemas conceptuales”, Revista Teorema, Vol. 23, \#3.

Siegel, H. (1988), Educating Reason. Rationality, critical thinking and education. Londres: Routledge.

(1997), Rationality redeemed? Further dialogues on an educational ideal. New York: Routledge.

(2010), "Critical Thinking", in Penelope Peterson, Eva Baker, Barry McGaw, (Editors), International Encyclopedia of Education, volume 6, pp. 141- 145. Oxford: Elsevier.

Skidelsky, L. (2011), “Autoconocimiento y Atribución de Estados Mentales en Teoría de la Mente", Revista Argentina de Ciencias del Comportamiento, Vol. 3, №1, 34-53.

Taney, J. (2009), "Rethinking Ryle. A Critical Discussion of The Concept of Mind", en Ryle, G. The concept of mind. London: Routledge.

Thayer-Bacon, B. (2000), "Transforming and Redescribing Critical Thinking", Inquiry: critical thinking across the disciplines. Summer, Vol. 19, №. 4. pp. 4-7.

Uhlmann, E.L., and Cohen, G.L. (2005), “Constructed criteria: Redefining merit to justify discrimination". Psychological Science 16, 474-480.

VerLinden, J. (2001), "Transforming Critical Thinking: Thinking Constructively", Inquiry: critical thinking across the disciplines. Summer, Vol. 20, №. 4.

Walters, K. (1994a), "Introduction: Beyond logism and critical thinking", New perspectives in critical thinking. New York, State of University of New York press, pp. 1-22.

(1994b), "Critical thinking, rationality, and vulcanization of students", New perspectives in critical thinking. New York, State of University of New York press, pp. 61-80. 